\title{
Nasal tuberculosis - an update of current clinical and laboratory investigation
}

\author{
L MASTERSON $^{1}$, I SROUJI $^{1}$, R KENT ${ }^{2}$, A P BATH ${ }^{3}$ \\ ${ }^{1}$ Department of ENT, Cambridge University Hospitals NHS Foundation Trust, ${ }^{2}$ Department of Microbiology, The \\ Ipswich Hospital NHS Trust, and ${ }^{3}$ Department of ENT, Norfolk and Norwich University Hospitals NHS Foundation \\ Trust, UK
}

\begin{abstract}
Objective: To report a case of primary nasal tuberculosis, and to discuss the diagnostic difficulties encountered.

Setting: A teaching hospital in Norwich, UK.

Method: Case report and review of the English language literature concerning tuberculosis affecting the head and neck region.

Result: The diagnosis of nasal tuberculosis is based on: histological identification of granulomatous inflammation; positive testing for acid-alcohol resistant bacilli; and positive culture. Newer diagnostic tests have the advantage of speed and improved accuracy, but are not as yet completely evaluated for the diagnosis of extra-pulmonary tuberculosis.

Conclusion: It is important to consider nasal tuberculosis in the initial differential diagnosis. The quest to exclude a malignancy may lead to unacceptable delays in treatment.
\end{abstract}

Key words: Nasal Cavity; Tuberculosis; Investigative Techniques; Nucleic Acid Amplification Test; Microscopy; Skin Tests; Histocytological Preparation Techniques

\section{Introduction}

We describe a case of primary nasal tuberculosis (TB) which presented a great diagnostic challenge, both clinically and in the laboratory. Primary nasal tuberculosis is an uncommon condition mainly confined to endemic areas of the world. ${ }^{1}$ However, over recent years the incidence of pulmonary and extra-pulmonary tuberculosis (including nasal TB) has risen in the UK. ${ }^{2}$ Extra-pulmonary tuberculosis in the ENT region mainly affects the cervical lymph node chain, with rarer manifestations in the larynx, nasopharynx, tonsils, salivary glands and middle ear. A previous review of the medical literature identified 35 reported cases of nasal TB, only one-third of which were due to primary infection without pulmonary involvement. ${ }^{3}$

\section{Case report}

A healthy, 79-year-old Caucasian woman presented with a six-month history of unilateral nasal obstruction, crusting and recurrent epistaxis.

Anterior rhinoscopy revealed friable mucosal oedema and abundant thick granulation tissue localised to the left side of the nasal septum (Figure 1). A full blood count, blood chemistry analysis, liver function test, anti-neutrophil cytoplasmic antibody test, chest X-ray and urinalysis were all within normal limits and did not suggest systemic disease. The erythrocyte sedimentation rate was raised, at $43 \mathrm{~mm} / \mathrm{hr}$ (normal level $<30 \mathrm{~mm} / \mathrm{hr}$ ).

A biopsy of the affected area under local anaesthesia showed inflammatory tissue with few histiocytes and no evidence of malignancy.
The patient had a poor response to a course of topical antibiotic cream preceded by nasal douching.

Three further biopsies were taken over the following 18 months; one showed non-caseating, granulomatous inflammation. An atypical mycobacterial infection was suspected, and so further surgery was carried out under general anaesthesia to formally excise the lesion, leaving the defect to heal via secondary intention. During the procedure, it was noted that a full thickness defect existed in the posterior septal cartilage.

Histological examination of this surgical specimen revealed (for the first time) caseating granulomatous change (Figure 2).

Modified Ziehl-Neelsen staining was negative, but a retrospective review and staining of previous samples revealed one to be positive for acid-alcohol resistant bacilli. Tissue was sent to the National Mycobacterium Reference Unit in London. There, a rapid polymerase chain reaction assay targeting Mycobacterium tuberculosis complex RNA polymerase $\beta$ chain was reported as negative; however, after 20 days of incubation, a culture on Lowenstein-Jensen medium showed growth of $M$ tuberculosis.

The diagnosis of primary nasal TB was made, 21 months after the patient's initial presentation.

The case was subsequently managed in cooperation with the local respiratory physician. Treatment with isoniazid, rifampicin, pyrazinimide and ethambutol was instigated.

After six months of therapy, a review of the patient revealed the nasal cavity to be dry and the lesion to have almost completely cleared, with no evidence of septal 


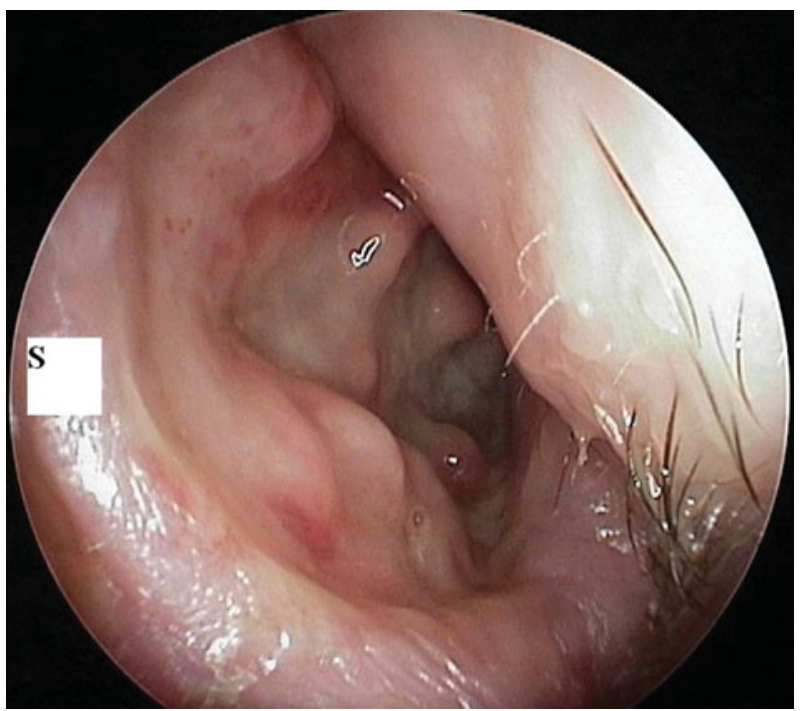

FIG. 1

Nasendoscopic view showing an irregular, friable, 'apple jelly' consistency mass. $\mathrm{S}=$ nasal septum

perforation (despite the previous operative findings of significant cartilage involvement).

\section{Discussion}

Tuberculosis is a chronic communicable disease in which the lungs are the prime target, although any organ may be infected. Human disease is mainly caused by $M$ tuberculosis but also, less commonly, by other members of the mycobacterium genus such as $M$ bovis. Although the overall incidence of tuberculosis has declined since the 1950s, there has been a recent increase in reported UK cases. This has been attributed to a rise in the number of immunocompromised patients and anti-TB drug resistant strains. ${ }^{4}$ The occurrence of extra-pulmonary TB is rising still further, and now constitutes 40 per cent of the total incidence of the disease, with many cases involving the ENT region. ${ }^{2}$

Primary nasal tuberculosis may be caused by inhalation of infected particles or traumatic digital inoculation. The majority of previously reported nasal TB cases were

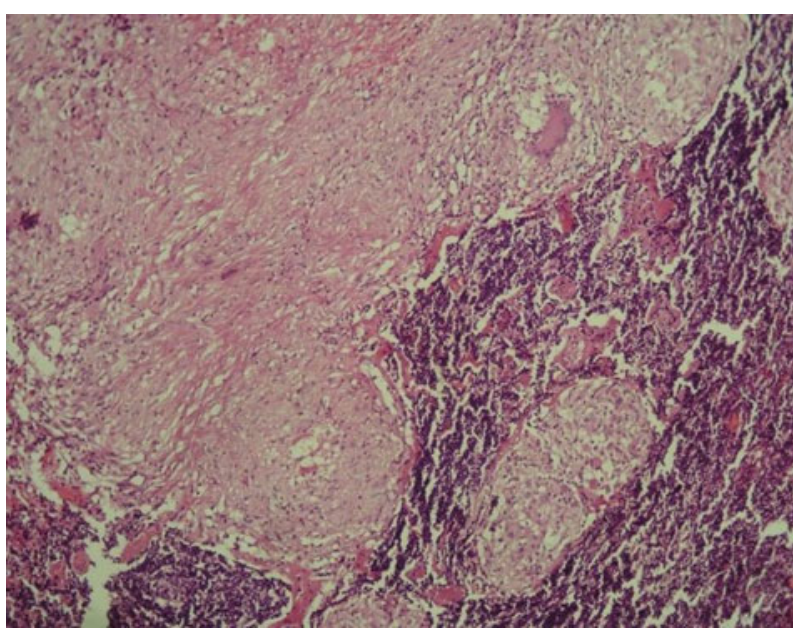

FIG. 2

Photomicrograph of surgical specimen showing tubercular caseous necrosis. $(\mathrm{H} \& \mathrm{E} ; \times 100)$ healthy before infection. ${ }^{5}$ The nasal septum is more frequently involved than the lateral wall; if the latter is affected, the inferior turbinate is the most common location. The appearance of a friable nasal mass and associated cervical lymphadenopathy may delay the true diagnosis as the quest for histological evidence of malignancy continues through repeated biopsies. In adults, additional differential diagnoses should include lethal midline granuloma, Wegener's granuloma, sarcoid, syphilis and fungal infection.

Treatment of nasal TB is usually straightforward, being similar to that for classical pulmonary TB. The British Thoracic Society guidelines call for a short initial course of bactericidal chemotherapy, followed by a prolonged sterilising regimen. ${ }^{6}$ However, as already demonstrated, the clinician's challenge lies in establishing the diagnosis, which is the subject of further discussion below.

\section{Histology}

Granulomatous lesions in the nasal cavity may represent either local disease or a manifestation of a systemic disorder. In either situation, it is paramount that the differential diagnosis includes malignancy; however, the focus on excluding this can lead to mycobacterial infection being overlooked as the cause.

A granuloma is an organised collection of macrophages (also termed histiocytes) arising as an inflammatory response to infection or a foreign body. It must be differentiated from granulation tissue, which is newly formed vascular tissue arising during normal wound healing. Histiocytes are nonspecific inflammatory cells, and were demonstrated in all of our patient's biopsies. Although they can represent a precursor to granuloma formation, their isolated presence is non-diagnostic.

The characteristic TB lesion is a spherical granuloma with central caseous necrosis. ${ }^{7}$ Granulomata caused by tubercle bacilli tend to have more giant cells and larger numbers of epithelioid cells than those caused by other diseases. ${ }^{8}$ In the presented case, biopsy of a non-caseating granuloma created confusion and contributed to the diagnostic delay. However, it is worth noting that such non-caseating granulomata can be precursors to the classic form seen in TB, and their mention in the pathology report should prompt the clinician to keep TB on the differential diagnosis list.

\section{Microscopy}

Tuberculosis microscopy employs the Ziehl-Neelsen stain, which shows the characteristic acid-fast mycobacterial cell wall composition and structure. The Ziehl-Neelsen stain was used in the present case, but the auramine-rhodamine fluorochrome technique is an alternative. Microscopic examination will confirm the presence of acid-fast bacilli, but does not always indicate the viability of the organisms or whether the pathogen is TB (as atypical mycobacteria will also stain positively). In general, regardless of which stain is used, the test is highly specific but poorly sensitive (with more than 98 per cent specificity versus 41-65 per cent sensitivity; see Table I). In addition, Ziehl-Neelsen staining fails to identify mycobacteria present in numbers of less than $10^{4}$ per ml; hence, many infections do not stain positively, leading to delayed diagnosis. ${ }^{10}$

\section{Routine culture}

During the extensive search for a malignant or inflammatory cause for our patient's nasal mass, microbial culture was only 
TABLE I

LABORATORY TESTS FOR EXTRA-PULMONARY TB

\begin{tabular}{lrrrlll}
\hline Test & Sens $(\%)$ & Spec $(\%)$ & Timescale & UK availability & Diffs atyp myco? & Comments \\
\hline Skin test $^{9}$ & 79 & 76 & $72 \mathrm{~h}$ & Primary care & No & Heaf test no longer used in UK \\
Microscopy $^{10}$ & $>98$ & $41-65$ & $1-2 \mathrm{~h}$ & DGH lab & No & ZN stain most common \\
Culture $^{11}$ & $46-63$ & 98 & $\sim 21 \mathrm{~d}$ & DGH lab & Yes & Faster results with liquid-based media \\
NAAT $^{10}$ & 78 & 96 & $<24 \mathrm{~h}$ & RMRL & Yes & High unit cost $(\sim £ 130)$ \\
Interferon $\gamma^{12}$ & 85 & 95 & $16-24 \mathrm{~h}$ & RMRL & Yes & Requires whole blood sample \\
\hline
\end{tabular}

$\mathrm{TB}=$ tuberculosis; sens $=$ sensitivity; spec $=$ specificity; diffs atyp myco $=$ differentiates atypical mycobacteria; $\mathrm{h}=$ hours; $\mathrm{DGH}=$ district general hospital; lab = laboratory; ZN = Ziehl-Neelsen; $\mathrm{d}=$ days; NAAT = nucleic acid amplification test; RMRL = regional mycobacterial reference laboratory

requested at a later stage, as is often the case with this clinical presentation. Tuberculosis infection should always be kept in mind when histological analysis is non-diagnostic, or for any friable tissue with an atypical macroscopic appearance.

Nasal secretions and swab specimens have a very low yield and should not be used to rule out nasal TB. ${ }^{13}$ In order to obtain a definitive diagnosis, a biopsy sample should be sent for staining and culture, in addition to routine histology, along with notification of the types of organism suspected. Traditional solid mycobacterial cultures may require up to three to six weeks' incubation to enable $M$ tuberculosis isolation; however, the use of automated, liquidbased systems can hasten growth detection (by 6 to 12 days). Pulmonary TB cultures have the highest sensitivity of currently available tests, with detection rates of up to 98 per cent cited. ${ }^{10}$ Such culture also permits species identification and drug sensitivity testing. In nasal TB, however, previous studies have shown that a large proportion of initial solid biopsy cultures and acid-fast bacilli staining turns out to be negative. ${ }^{13}$ One reason for this is the comparative paucity of tubercular bacilli in extra-pulmonary sites, with culture negativity rates of 50-75 per cent. ${ }^{11}$

\section{Nucleic acid amplification tests}

Molecular tests are able to detect minute amounts of bacterial genetic material (either DNA or RNA target sequences), whether the bacteria is alive or dead. If the target organism is present in the sample, amplification of representative genetic material will usually be possible. A variety of techniques are available, including amplification of the target nucleic acid (e.g. using polymerase chain reaction) and amplification of a nucleic acid probe (e.g. ligase chain reaction). These techniques allow identification within 24 hours, but are costly and limited to specialist centres. A recent review of the diagnosis of extra-pulmonary TB suggested that the best commercial nucleic acid amplification tests are highly specific for the disease (at 96 per cent) but only moderately sensitive (78 per cent). ${ }^{7}$

\section{Immunological tests}

Tuberculin skin tests (e.g. Mantoux or Heaf) are the most widely available method for diagnosing tuberculous infection in the absence of active disease (i.e. latent infection). The Heaf test was discontinued in the UK in 2005. The Mantoux test involves an intradermal injection of a standard purified tuberculin protein derivative. The response is measured as the amount of induration after two to three days. Interpretation of skin testing depends on the size of the indurated area and the patient's age and risk factors. The test result is significant if more than $5 \mathrm{~mm}$ in patients with immunodeficiency (e.g. due to human immunodeficiency virus infection or organ transplantation), more than $10 \mathrm{~mm}$ in patients with intermediate risk (e.g. close contacts of TB patients, advanced haematological malignancy patients or immigrants from high prevalence communities), or more than $15 \mathrm{~mm}$ in individuals with no obvious risk factors. In this latter group, induration of $6-15 \mathrm{~mm}$ is likely to be due to Bacille Calmette-Guérin (BCG) vaccination, or to exposure to atypical mycobacteria which can produce a false positive result. ${ }^{9}$

\section{- Primary nasal tuberculosis (TB) is rare}

- The UK incidence of extra-pulmonary TB is rising

- Nasal TB can mimic a malignancy, and the quest to exclude the latter may cause unacceptable treatment delays

- Diagnosis is based on the histological finding of granulomatous inflammation, positive testing for acid-alcohol resistant bacilli, and positive culture

- New diagnostic techniques include polymerase chain reaction and interferon $\gamma$ assay; both are quicker than previous methods, but are not yet fully evaluated for head and neck TB

Interferon $\gamma$ assays (Quantiferon ${ }^{\circledR}$-TB or T-spot $\left.{ }^{(}\right)$) have been developed to replace tuberculin skin tests, in order to detect latent TB infection. Blood is taken from the patient and incubated with antibodies specific for mycobacterial antigens present in TB complex strains but absent from BCG vaccine strains. As these rapid tests detect the organism directly and do not rely on an immune response, they require only one patient visit and are not susceptible to interpretation variations or observer error. They may have a future role in the detection of active TB. ${ }^{10}$

\section{Conclusion}

Primary nasal tuberculosis is a rare finding. However, the symptoms of nasal tuberculosis can mimic those of malignancy, and a biopsy must always be taken. Diagnosis is based on the histological identification of granulomatous inflammation, positive testing for acid-alcohol resistant bacilli, and positive culture. Polymerase chain reaction amplification and modern immunological methods have the advantages of more rapid diagnosis and good performance, albeit less consistently than in pulmonary TB cases. Infective causes form a large part of the differential diagnosis of atypical nasal soft tissue masses, and for this reason we recommend routine microbiology (including microscopy 
and culture) plus TB culture in all such cases. These investigations are easily overlooked as the clinician seeks a malignant cause for their patient's condition.

\section{Acknowledgements}

We thank Professor F Drobniewski and $\mathrm{Dr} T$ Brown (National Mycobacterium Reference Laboratory, Health Protection Agency, London) for their assistance in the production of this article.

\section{References}

1 Raviglione M, Uplekar M. WHO's new Stop TB Strategy. Lancet 2006;367:952-5

2 Anderson C, Moore J, Kruijshaar M, Abubakar I. Tuberculosis in the UK: Annual Report on Tuberculosis Surveillance in the UK 2008. London: Health Protection Agency, 2008

3 Butt A. Nasal tuberculosis in the $20^{\text {th }}$ century. Am J Med Sci 1997;313:332-5

4 World Health Organization. Global Tuberculosis Control: Epidemiology, Strategy, Financing: WHO Report. Geneva: World Health Organization, 2009

5 Yong MK, Kim AH, Park YH, Kim DH, Rha K. Eight cases of nasal tuberculosis. Otolaryngol Head Neck Surg 2007;137: $500-4$

6 Ormerod L. The evidence based treatment of tuberculosis: where and why are we failing? Thorax 2008;63:388-90

7 Rubin R, Strayer D, eds. Rubin's Pathology: Clinicopathologic Foundations of Medicine, 5th edn. Philadelphia: Lippincott Williams \& Wilkins, 2007
8 Waldman SR, Levine HL, Sebek BA, Parker W, Tucker HM. Nasal tuberculosis: a forgotten entity. Laryngoscope 1981;91: $11-16$

9 Starke J. Tuberculosis skin testing: new schools of thought. Pediatrics 1996;98:123-5

10 Dinnes J, Deeks J, Kunst H, Gibson A, Cummins E, Waugh N et al. A systematic review of rapid diagnostic tests for the detection of tuberculosis infection. Health Technol Assess 2007;11: 23-53

11 Oberoi A, Aggarwal A. Comparison of the conventional diagnostic techniques, BACTEC and PCR. J Med Ed Res 2007;9: 179-81

12 Dosanjh DP, Hinks TS, Innes JJ, Pasvol G, Hackforth S, Varia H et al. Improved diagnostic evaluation of suspected tuberculosis. Ann Intern Med 2008;148:325-36

13 Goguen LA, Karmody CS. Nasal tuberculosis. Otolaryngol Head Neck Surg 1995;113:131-5

Address for correspondence:

Mr Liam Masterson,

Department of ENT,

Cambridge University Hospitals NHS Foundation Trust,

Cambridge CB2 0QQ, UK

Fax: + 44 (0) 1223217559

E-mail:1mm398@doctors.org.uk

Mr L Masterson takes responsibility for the integrity of the content of the paper

Competing interests: None declared 